\title{
A Relatividade Geral e a Sonda Gravidade B
}

DOI: https://doi.org/10.35168/2176-896X.UTP.Tuiuti.2020.Vol7.N62.pp121-139 em Ensino de Física na Universidade Regional do Cariri - URCA. Graduação em Física pela Universidade Estadual do Sudoeste da Bahia, mestrado em Física e doutorado em Física pela Universidade Federal do Rio Grande de Norte . 


\section{A Relatividade Geral e a Sonda Gravidade B}

\section{Resumo}

Em 1918, Hans Thirring, usando a aproximação de campo fraco para as equações de campo de Einstein, deduziu que uma casca massiva girando lentamente arrasta os referenciais inerciais no sentido de sua rotação. No mesmo ano Joseph Lense aplicou na Astronomia os cálculos de Thirring. Posteriormente, este efeito ficou conhecido como efeito Lense-Thirring. Juntamente com o efeito de Sitter, esses fenômenos foram recentemente testados através de giroscópios em órbita em torno da Terra, uma antiga proposta feita por George E. Pugh em 1959 e por Leonard I. Schiff em 1960. O satélite da GPB foi lançado em órbita em torno da Terra a uma altitude de $642 \mathrm{~km}$ pela NASA em 2004. Resultados apresentados em maio de 2011 mostram claramente a existência do efeito Lense-Thirring e do efeito de Sitter com precisão de 19\% e de $0,28 \%$ respectivamente. Esses resultados estão em bom acordo com os valores previstos pela teoria da Relatividade Geral. Neste artigo busca-se abordar de forma breve e didática alguns elementos fundamentais para a compreensão da relatividade geral, a história da missão Sonda Gravidade B e os conceitos físicos que deram suporte as ideias para a elaboração do projeto desta missão.

Palavras-chave: Gravidade. Rotação. Terra. 


\section{The general relativity end the Gravity Probe B}

\section{Abstract}

In 1918 Hans Thirring, using the weak field approximation to the Einstein's field equations, deduced that a slowly rotating massive shell drags the inertial frames in the direction of its rotation. In the same year, Joseph Lense applied to astronomy the calculations of Thirring. Later, that effect came to be known as the Lense-Thirring effect. Along with the de Sitter effect, those phenomena were recently tested by a gyroscope in orbit around the Earth, as proposed by George E. Pugh in 1959 and Leonard I. Schiff in 1960. The GPB satellite was launched into orbit around the Earth at an altitude of $642 \mathrm{~km}$ by NASA in 2004. Results presented in May 2011 clearly show the existence of the Lense-Thirring effect and de Sitter effect measured with an accuracy of $19 \%$ and of $0.28 \%$ respectively. These results are in a good agreement with the General Relativity predictions. This paper seeks to briefly and didactically address some fundamental elements for the understanding of general relativity, the history of the Gravity B probe mission and the physical concepts that supported the ideas for the elaboration of this mission project.

Keywords: Gravity. Rotation. Earth. 


\section{A Relatividade Geral e a Sonda Gravidade B}

\section{Introdução}

A teoria de gravidade mais aceita, atualmente, é a relatividade geral, proposta por Albert Einstein em 1915. A relatividade geral é uma teoria geométrica do campo gravitacional cujo desenvolvimento surgiu através de reflexões sobre o conceito de inércia bem como do desejo de compatibilizar a teoria da Relatividade Restrita com a gravitação newtoniana (FALCIANO, 2009; CASTINEIRAS e CRISPINO, 2019). Na teoria de Einstein, o campo gravitacional é descrito por um tensor métrico constituído de 16 componentes, das quais 10 são independentes e são funções das coordenadas espaciais e do tempo.

A dinâmica da relatividade geral é descrita por um conjunto de equações que relacionam as propriedades geométricas do espaço-tempo, descritas por uma métrica e por suas derivadas de primeira e de segunda ordem, com o tensor energia-momento. O tensor energia-momento é a generalização dos conceitos de energia e momento de uma partícula para a descrição de campos. É por meio desse tensor que a distribuição de matéria é descrita (HARTLE, 2003).

A princípio, qualquer métrica pode ser solução das equações de Einstein. Entretanto, para que tenhamos soluções exatas, com interpretação física, precisamos impor restrições ao tensor energiamomento. Por essa razão, as soluções exatas restringem-se a um pequeno grupo que permite que as propriedades físicas do espaço-tempo sejam descritas sem recorrer à integração numérica de equações diferenciais de segunda ordem.

No século passado a teoria de Einstein foi alvo de muitos testes experimentais e, até o momento, no regime de campo fraco, ela apresenta resultados satisfatórios. Em particular, em 2011, as predições de Einstein quanto à rotação da fonte do campo gravitacional foram confirmadas por mais um teste no sistema solar. Trata-se do experimento Sonda Gravidade B que forneceu medidas, com precisão satisfatória, dos efeitos de Sitter e do efeito Lense-thirring. O efeito de Sitter refere-se à curvatura do espaço-tempo criada pela presença de uma distribuição de massa enquanto o efeito 


\section{A Relatividade Geral e a Sonda Gravidade B}

Lense-Thirring é observado quando um corpo teste está submetido ao campo gravitacional criado por uma distribuição de massa em rotação.

Embora a relatividade geral seja a teoria de gravidade mais aceita na atualidade, a sua compreensão é pouco expressiva por parte do público externo e interno a instituições de ensino superior ou médio. É possível apontar, no mínimo, duas justificativas para esta afirmação. Uma delas é o formalismo matemático avançado utilizado para estudar a relatividade geral e a outra, é o número reduzido de referências que abordem o tema de forma mais conceitual e didática. Neste artigo busca-se abordar de forma breve e didática alguns elementos fundamentais para a compreensão da relatividade geral, a história da missão Sonda Gravidade B e os conceitos físicos que deram suporte as ideias para a elaboração do projeto desta missão.

\section{A teoria de gravidade de Einstein}

Na relatividade geral, a gravidade surge como uma manifestação da curvatura do espaço-tempo. A interação entre a matéria e o espaço-tempo é tal que a matéria curva o espaço-tempo, e este por sua vez define a trajetória de uma partícula de acordo com a sua estrutura geométrica. Apesar de brilhante e inovadora, a relatividade geral possui um formalismo matemático extremamente complexo e, por essa razão, a sua difusão para o público leigo é ainda bastante tímida. Entretanto, é possível compreender a base teórica da relatividade geral a partir de três princípios básicos (LENSE, POMPEIA, STUDART, 2019; CASTINEIRAS e CRISPINO, 2019):

\footnotetext{
- Princípio Geral da Relatividade: as leis da Física devem ser as mesmas em referenciais inerciais ou não inerciais.

- Princípio da Covariância Geral: as leis da Física devem permanecer inalteradas para transformações de coordenadas gerais.
} 


\title{
A Relatividade Geral e a Sonda Gravidade B
}

\begin{abstract}
- Princípio da Equivalência: em cada ponto do espaço-tempo é possível estabelecer um sistema de referência localmente inercial, no qual os princípios da relatividade restrita são válidos.
\end{abstract}

Existe uma variedade de soluções exatas que têm desempenhado um papel muito importante no desenvolvimento tanto teórico como experimental da relatividade geral (LETELIER, 2005). No entanto, problemas como a descrição de um universo inhomogêneo (a nível cosmológico), o campo gravitacional de uma estrela em rotação, o problema de dois corpos e a propagação de radiação gravitacional estão sem soluções exatas (KRAMER, 1980). Uma das soluções exatas, talvez a mais importante, das equações de Einstein é a solução que representa o exterior de uma distribuição de matéria-energia com simetria esférica. Embora os sistemas com simetria esférica sejam razoavelmente simples, eles são fisicamente relevantes. Isso se deve ao fato de que muitos objetos estudados na escala astronômica são aproximadamente esféricos.

A solução que descreve o espaço-tempo em torno de uma distribuição de matéria esfericamente simétrica em repouso foi obtida por Karl Schwarzschild em 1916, poucos meses depois de Einstein publicar suas equações de campo para o vazio. A solução de Schwarzschild está na base dos três testes clássicos da relatividade geral, quais sejam, o deslocamento de linhas espectrais pela presença do campo gravitacional, o desvio de um feixe de luz que passa perto de uma estrela e a precessão do perihélio de Mercúrio. Além disso, esta solução está intimamente ligada a uma das previsões mais famosas da Relatividade Geral, que é a possível existência de buracos negros.

As chamadas soluções de Einstein-Rosen, que representa uma onda com simetria cilíndrica, também são bastante relevantes para a relatividade geral. Existe também uma linha de soluções usadas no contexto da Cosmologia e, nessa linha, utiliza-se as soluções de Friedman-LemaîtreRobertson-Walker para descrever um Universo homogêneo e isotrópico em expansão. 


\section{A Relatividade Geral e a Sonda Gravidade B}

As soluções que descrevem o campo gravitacional de um corpo com simetria axial representam um papel fundamental, tanto na teoria da gravitação de Newton, como na teoria da gravitação de Einstein. Isso se deve ao fato de que a forma natural de um fluido isolado auto gravitante tem simetria axial (LETELIER, 2005). Na prática, tais fluidos realizam movimentos de rotação e para estudá-los de forma mais profunda é necessário recorrer às soluções de Kerr (KERR, 1963). A distribuição de matéria representada pela métrica de Kerr gira com uma velocidade angular constante. $\mathrm{O}$ espaço-tempo em torno de um buraco negro em rotação é representado pela solução de Kerr e sua forma e tamanho dependem apenas de sua massa e de sua rotação. Se não houver rotação, a solução é idêntica à de Schwarzschild. Apesar de muitas tentativas e alguns resultados parciais, ninguém conseguiu adaptar a métrica de Kerr a uma solução válida para o interior de uma distribuição de matéria em rotação. Se qualquer solução interior existir, deve ser uma solução na qual a velocidade angular é suficientemente pequena para que termos de segunda ordem ou de ordem superior sejam desprezados (COHEN, 1967).

\section{A analogia entre a gravitação e o eletromagnetismo}

A analogia entre a Lei da gravitação de Newton e a Lei de Coulomb da Eletricidade levou a uma descrição da teoria da gravitação de Newton em termos de um campo gravitoelétrico. Posteriormente, com o desenvolvimento do Eletromagnetismo, a descrição desta teoria não se limitou apenas a campos gravitoelétricos, mas também aos chamados campos gravitomagnéticos (IORIO et. al., 2011; RUGGIERO, 2002; TARTAGLIA, 2004; MALEKOLKALAMI, 2009; MASHHOON, 2006; TSOUBELIS, 1987). O gravitomagnetismo é um fenômeno que consiste na interação gravitacional gerada pelo movimento de rotação de uma massa, da mesma forma que os fenômenos magnéticos são gerados a partir do movimento de cargas elétricas.

O efeito gravitomagnético mais famosos é o efeito Lense-Thirring que, por sua vez, é observado quando consideramos um corpo teste no campo de uma distribuição de matéria em rotação. Esse 


\section{A Relatividade Geral e a Sonda Gravidade B}

efeito foi derivado pela primeira vez, em 1918, pelos físicos austríacos Hans Thirring e Josef Lense e por isso ficou conhecido como efeito Lense-Thirring. Lense e Thirring previram a precessão do eixo de um giroscópio próximo a um corpo em rotação, por exemplo, a Terra.

No contexto da relatividade geral, o efeito Lense-Thirring não é o único efeito relevante quando estudamos um giroscópio nas proximidades de uma distribuição de massa em rotação. Uma distribuição de massa, independente do seu movimento de rotação, provoca uma curvatura no espaço-tempo e, como consequência, o eixo de um giroscópio nas suas proximidades não será mais paralelo a sua orientação inicial ao completar um círculo. Este efeito é conhecido como efeito de Sitter. Em particular, o sistema Terra-Lua pode ser considerado como um único corpo, que se move no campo gravitacional do Sol. Para o sistema Terra-Lua, o efeito de Sitter foi testado experimentalmente por Bertotti, Ciufolini e Bender em 1987, com uma precisão de aproximadamente 10\% (CIUFOLINI e WHEELER, 1995); por Shapiro et. al. em 1988, com uma precisão de 2\% (SHAPIRO et al. 1988) e Williams et al. em 1996 com uma precisão de aproximadamente 1\% (WILLIAMS, NEWHALL e DICKEY, 1996).

É importante enfatizar que o efeito Lense-Thirring não é previsto pela gravitação newtoniana.

\section{A Sonda Gravidade B}

A Sonda Gravidade B - Gravity Probe B (GPB) - é uma missão desenvolvida pela Universidade de Stanford e pela NASA (National Aeronautics and Space Administration), para testar, com uma precisão jamais obtida, os seguintes efeitos previstos pela relatividade geral de Einstein:

- O efeito de Sitter- devido à curvatura do espaço-tempo criada pela presença de um corpo massivo, como a Terra.

Revista Tuiuti: Ciência e Cultura, v.7 n.62. p. 121-139, Curitiba, 2021 


\section{A Relatividade Geral e a Sonda Gravidade B}

- O efeito Lense-Thirring- devido ao arrastamento do espaço-tempo em consequência da rotação da Terra.

O GPB é o primeiro experimento que tem como um dos objetivos principais medir o efeito Lense-Thirring que é um efeito extremamente pequeno para objetos do nosso sistema solar e medi-lo é uma tarefa que exige métodos precisos. No nosso sistema solar os efeitos relativísticos são pequenas correções da teoria newtoniana e consequentemente, medir o efeito Lense-Thirring em meio a uma série de efeitos clássicos, é muito difícil (STELLA e POSSENTI, 2010; BARKER e O'CORNNEL, 1970).

\subsection{0 projeto GPB}

A ideia de testar a relatividade geral com giroscópios em órbita foi sugerida independentemente por George Pugh, em 1959, e por Leonard Schiff, em 1960. Na época, Leonard Schiff era coordenador do Departamento de Física da Universidade de Stanford e, achou por bem discutir suas ideias com dois colegas do Departamento de Física, os professores William Little e William Fairbank.

Havia chegado há pouco tempo na Universidade de Stanford, no Departamento de Aeronáutica, o professor Robert Cannon o qual tinha experiência considerável com a mecânica de giroscópios e pretendia montar um laboratório no Departamento de Aeronáutica e Astronáutica em Stanford. Após uma reunião com Cannon, Fairbank e Schiff, surgiu a colaboração essencial dos dois departamentos para a realização do teste espacial da relatividade geral. Em janeiro de 1961, Fairbank e Schift enviaram uma carta de três páginas para o Instituto de Ciências Espaciais (Office of Space Sciences) da NASA, propondo um possível experimento espacial para testar a Relatividade Geral usando giroscópios. 


\section{A Relatividade Geral e a Sonda Gravidade B}

Durante o primeiro ano de Nancy Roman como chefe do Programa de Astronomia Espacial da NASA, o Instituto de Ciências havia recebido muitas propostas de testes espaciais, inclusive a proposta de Fairbank e Schiff. Roman e outros pesquisadores da NASA estavam ansiosos para apoiar tal experimento, mas era necessário físicos e engenheiros especialistas neste campo. Por iniciativa de Roman, uma conferência sobre testes espaciais patrocinada pela NASA, foi realizada na Universidade de Stanford de 20 a 21 de julho de 1961. Além de um grupo de físicos e de engenheiros, Schiff, Cannon e dois estudantes de pós-graduação, o Daniel Debra e o Benjamin Lange, participaram da conferência. Daniel Debra estava concluindo o doutorado, e Benjamin Lange havia ingressado recentemente no curso de pós-graduação. Ambos eram alunos de Cannon.

Em 1962, Fairbank encontrou Francis Everitt, que na época era um pesquisador da Universidade da Pensilvânia. Fairbank incentivou Everitt a ingressar na Universidade de Stanford como primeiro membro a se dedicar exclusivamente ao teste espacial da relatividade geral. Durante este período, Fairbank e Cannon começaram a elaborar uma proposta mais detalhada para a NASA, a fim de obter o financiamento para o desenvolvimento de um satélite que orbitasse a Terra, em queda livre, sem sofrer os efeitos da pressão de radiação solar ou pressão atmosférica. Fairbank convidou Everitt para ajudar a elaborar essa proposta, a qual foi apresentada para a NASA em novembro de 1962. Fairbank e Cannon foram designados como pesquisadores principais desta proposta e, Schiff como um assessor. A proposta foi aceita e financiada pela NASA em novembro de 1963. Em 1966, Cannon deixou Stanford e Debra foi promovido para o cargo de pesquisador principal da área de Aeronáutica e Astronáutica. Debra atualmente é professor da Universidade de Stanford e continua como pesquisador principal do Departamento de Aeronáutica e Astronáutica. O teste do redshift gravitacional e este teste espacial foram denominados, Sonda Gravidade A e Sonda Gravidade B, respectivamente, em 1971 pela NASA. 


\section{A Relatividade Geral e a Sonda Gravidade B}

Em 1977, a equipe do GPB acreditava que a parte teórica do experimento estava concluída e, a NASA em 1980, convocou uma equipe para avaliar a estrutura tecnológica do GPB. Essa análise é chamada de fase A do estudo do GPB.

Em 1981, Francis Everitt havia se tornado a pessoa mais informada sobre todos os aspectos do experimento GPB. Por esse motivo ele foi promovido para cargo de pesquisador principal na área da Física e continuou nesse cargo até a divulgação dos resultados finais do GPB. Animados com os resultados da fase A, a NASA e a Universidade de Stanford realizaram um estudo muito mais detalhado, em 1982, chamado de fase B.

O custo para realizar o experimento era muito alto e inaceitável. Por essa razão, em 1983, Everitt, a NASA e a equipe da Universidade de Stanford reestruturaram o projeto, reduzindo o custo total do GPB sem sacrificar seus objetivos iniciais.

Em 1994 todo o equipamento para colocar o experimento em prática estava pronto e, o mês previsto para o lançamento do satélite seria outubro de 2000. Entretanto os anos seguintes representaram um período crítico para o GPB e devido a alguns problemas inesperados, como a condução de calor dentro e fora da sonda, o mês do lançamento foi adiado para abril de 2004.

Um fato curioso na história do GPB é que, por alguma razão, George Pugh não foi convidado para participar da Conferência de 1961, embora sua proposta de 1959, de testar a relatividade geral usando giroscópio tenha sido lida por Schiff na época. Apesar de Cannon e seus alunos estarem cientes da proposta de Pugh, devido às referências de Schiff, nenhum deles realmente leu ou viu a proposta até 1990. Aparentemente, Francis Everitt foi o primeiro membro da equipe do GPB, com exceção de Schiff, que realmente leu a proposta de Pugh. Em 1989, após ministrar uma palestra sobre o GPB, Everitt recebeu uma cópia do artigo de Pugh. Após a leitura, Everitt parabenizou o autor e o convidou para participar de uma festa de aniversário de Debra, em julho de 1990. Durante a festa de Debra, Pugh ministrou uma palestra sobre a sua proposta de 1959 para toda a equipe do 


\section{A Relatividade Geral e a Sonda Gravidade B}

GPB. Assim, em 1990, a proposta de Pugh foi finalmente distribuída e lida por outros membros da equipe do GPB.

O GPB é o segundo experimento da NASA cuja finalidade era testar a relatividade geral. O primeiro, Sonda Gravidade A, estudou o efeito do campo gravitacional sobre as medidas de tempo usando relógios de hidrogênio. Esse experimento, chamado de redshift gravitacional, foi o primeiro teste da gravidade proposto por Einstein e é conhecido como um dos três testes clássicos da Relatividade Geral. A existência do redshift gravitacional decorre do princípio da equivalência, de modo que um relógio em um campo gravitacional é indistinguível de outro relógio idêntico em um referencial acelerado. A primeira medida precisa do redshift gravitacional, com $1 \%$ de precisão, foi realizada por Robert Pound e Glen Rebka, em 1960. Eles usaram dois relógios atômicos em movimento vertical na torre da Universidade de Havard. O teste do redshift gravitacional mais preciso foi realizado por Vessot et al. em 1976 (VESSOT et al, 1980).

A Sonda Gravidade A comparou o tempo decorrido em dois relógios de hidrogênio idênticos, um na Terra e o outro viajando por aproximadamente duas horas em um foguete. O satélite da Sonda Gravidade A foi lançado no dia 18 de junho de 1976 a uma altitude de $10.000 \mathrm{~km}$ em uma trajetória aproximadamente vertical. Esse experimento confirmou a previsão do redshift gravitacional com uma precisão de $0,02 \%$.

\subsection{0 lançamento do satélite do GPB}

O satélite da Sonda Gravidade B foi lançado por um foguete Delta II em 20 de abril de 2004, da Base militar de Vandenberg, depois de mais de 44 anos de preparação. A missão GPB consiste de um satélite em órbita polar em torno da Terra a uma altura de $642 \mathrm{~km}$. A órbita precisa ser alta o suficiente para que o satélite esteja quase livre da atmosfera terrestre, mas próxima da Terra o 


\section{A Relatividade Geral e a Sonda Gravidade B}

suficiente para que os efeitos gravitomagnéticos sejam observados no satélite. O satélite do GPB carrega quatro giroscópios e um telescópio. O telescópio e o eixo de rotação de cada giroscópio foram alinhados com um sistema de referência por um ano. Em seguida, foram realizadas as medidas das mudanças do eixo de rotação de cada giroscópio no plano da órbita (precessão de Sitter) e perpendicular ao eixo de rotação da Terra (precessão Lense-Thirring).

Os quasares constituem um sistema de referência adequado para medir as variações no eixo de rotação dos giroscópios do GPB. Isso acontece porque os quasares estão a uma distância muito grande da Terra e, consequentemente suas posições são consideradas fixas. A luminosidade dos quasares é demasiadamente fraca para qualquer telescópio óptico. Assim, o telescópio é alinhado com uma estrela próxima e mais brilhante cujo movimento pode ser mapeado em relação ao quasar. Os quasares (Quasi Stellar Radio Sources) foram descobertos em 1961, como intensas fontes de rádio, com aparência ótica aproximadamente estelar, azuladas. Edwin Ernest Salpeter (1925-2008) e Yakov Borisovich Zel'dovich (1914-1989) propuseram em 1964 que os quasares seriam, provavelmente, galáxias com buracos negros fortemente ativos no centro. Os quasares são objetos extremamente compactos e luminosos, emitindo mais energia do que centenas de galáxias juntas (KEPLER, 2004). Entre as 1.400 estrelas analisadas, a IM Pegasi (HR8703) foi a escolhida como a estrela guia do GPB. A IM Pegasi é um sistema binário (HR 8703 e HD 216489) situado na constelação de Pegasus. A IM Pegasi (HR 8703) é uma estrela gigante tipo K, (a sua massa é 1,8 vezes a massa solar) e temperatura de $4.800 \mathrm{~K}$. A segunda estrela é tipo $\mathrm{G}$ e sua massa é da ordem da massa solar. A IM Pegasi (HR8703) está situada na nossa galáxia à uma distância de aproximadamente 300 anos-luz da Terra. Como a IM Pegasi é uma fonte de rádio, seu movimento próprio é monitorado pela tecnologia do telescópio de rádio, de modo que a variação no eixo de rotação dos giroscópios não é afetada pelo seu movimento próprio.

Os giroscópios do GPB são esferas com 3,81 cm de diâmetro que são mantidas em uma câmara evacuada dentro de um tanque com 2.440 litros de hélio a -271,4 graus Celsius. Para conhecer e 


\section{A Relatividade Geral e a Sonda Gravidade B}

acompanhar a orientação do eixo de rotação dos giroscópios o grupo da Universidade de Stanford se apoiou no fenômeno conhecido como momento London que é o momento magnético de um supercondutor em rotação com relação a um sistema de referência inercial é conhecido como momento magnético London. Quando uma esfera de metal supercondutor gira, ela cria ao seu redor um campo magnético. O eixo do campo magnético se alinha perfeitamente com o eixo de rotação da esfera. Para observar o momento London nos giroscópios foi necessário revesti-los com uma fina camada de um metal supercondutor. O metal utilizado foi o nióbio o qual tornase supercondutor a uma temperatura de -263 graus Celsius. Assim, quando os giroscópios giram, criam um campo magnético cujo eixo coincide com o eixo de rotação. Portanto, para saber para qual direção o giroscópio aponta, é necessário monitorar a orientação do campo magnético. Isto é feito por um aparelho chamado SQUID (Superconducting Quantum Interference Device) - dispositivo de interferência quântica - que é ligado ao giroscópio por um aro fino que o enlaça. Quando o eixo do giroscópio se inclina, o momento London do campo magnético se inclina também e o SQUID detecta essa variação na direção do campo magnético. O SQUID é capaz de detectar até uma variação de $5^{-14}$ gauss.

O objetivo do experimento GPB era medir o efeito de Sitter com uma precisão de 0,01\% e o efeito Lense-Thirring com 0,1\% (EVERITT et. al., 2009). O experimento GPB testou estes dois efeitos medindo precisamente a precessão de quatro giroscópios em órbita em torno da Terra por um período de um ano e comparou os resultados experimentais com os resultados previstos pela relatividade geral.

\subsection{0 s resultados do GPB}

Em 14 de abril de 2007, após 18 meses de análises dos dados, os primeiros resultados do experimento GPB foram apresentados. O efeito de Sitter era claramente visível nos giroscópios 


\section{A Relatividade Geral e a Sonda Gravidade B}

GPB, confirmando as previsões de Einstein com uma precisão da ordem de 1,5 \% (EVERITT, 2009). A equipe da GPB explicou que os desvios dos resultados esperados surgiram em virtude dos torques produzidos nos giroscópios. Os resultados apresentados em setembro de 2009 mostraram claramente a existência do efeito Lense-Thirring, o qual foi medido com uma precisão de aproximadamente $14 \backslash \%$. Os resultados de 2009 mostraram a existência do efeito de Sitter com uma precisão superior ao apresentado em 2007 (EVERITT, 2009). Os resultados finais, apresentados na tabela (1), foram divulgados no dia 4 de maio de 2011 e o efeito Lense-Thirring foi então medido com uma precisão de aproximadamente 19\%, enquanto o efeito de Sitter com aproximadamente 0,28 \% de precisão (EVERITT et. al., 2011; WILL, 2011). A tabela (1) apresenta os resultados individuais dos quatro giroscópios combinados e o valor teórico previsto pela relatividade geral.

Tabela 1 - Tabela de resultados do experimento sonda gravidade B

Fonte Efeito de Sitter (msa/ano)

Efeito Lense-Thirring (msa/ano)

\begin{tabular}{c|cc}
\hline Giroscópio 1 & $6.588,6 \pm 31,7$ & $41,3 \pm 24,6$ \\
Giroscópio 2 & $6.707,0 \pm 64,1$ & $16,1 \pm 29,7$ \\
Giroscópio 3 & $6.610,5 \pm 43,2$ & $25,0 \pm 12,1$ \\
Giroscópio 4 & $6.588,7 \pm 33,2$ & $49,3 \pm 11,4$ \\
Média & $6.601,8 \pm 18,3$ & $37,2 \pm 7,2$ \\
Previsão Teórica & $6.606,1$ & 48,0
\end{tabular}

Fonte: Everitt et. al. (2011). 


\section{A Relatividade Geral e a Sonda Gravidade B}

As duas precessões previstas pela relatividade geral, para o giroscópio em relação à estrela guia IM Pegasi (HR8703) estão representadas esquematicamente na figura (1).

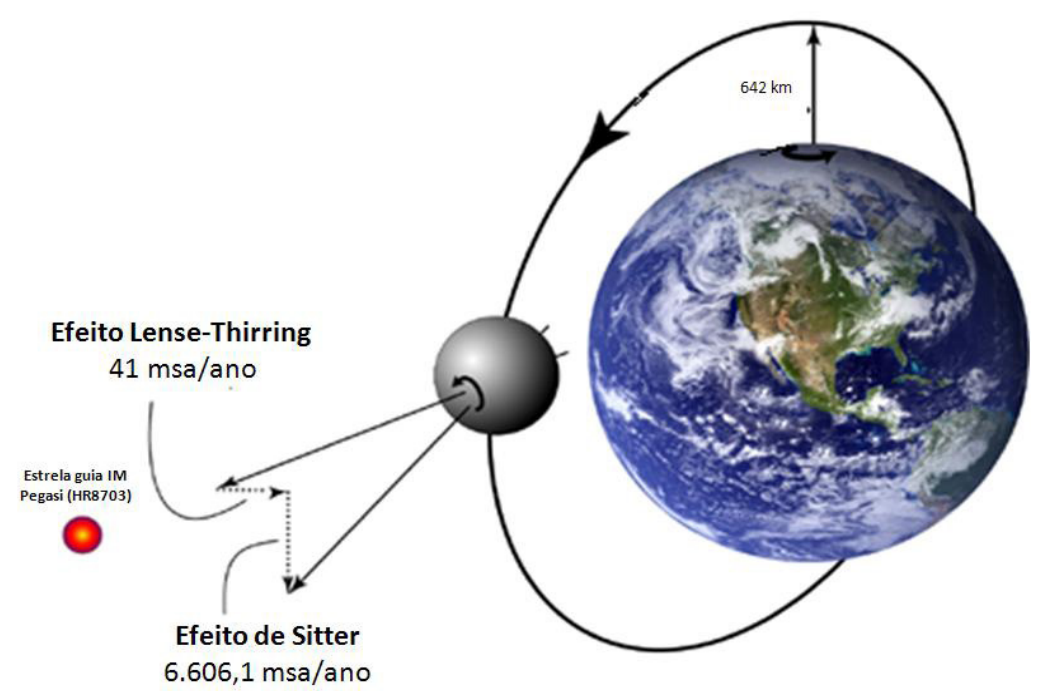

Figura 1 - Os dois tipos de precessão de um giroscópio em torno da Terra.

Fonte: Everitt et al. (2009).

\section{Considerações finais}

A relatividade geral é considerada uma teoria padrão, que descreve as estruturas astrofísicas e todo o universo. Entretanto, algumas inconsistências, relativas à curva de rotação das galáxias, a 


\section{A Relatividade Geral e a Sonda Gravidade B}

discrepância na massa dos aglomerados de galáxias e a expansão acelerada do Universo, sugerem fortemente que a gravidade de Einstein precisa ser revisada ou no mínimo estendida em algumas escalas. Tendo como base tais inconsistências, alguns pesquisadores optam por estudar teorias de gravidade alternativas a de Einstein. As teorias de gravidade alternativas são um novo paradigma da Física Moderna.

Embora o experimento da Sonda Gravidade B não tenha alcançado seu objetivo inicial, ela comprovou as previsões de Einstein no Sistema Solar, com precisão relativamente satisfatória, tendo em conta que os efeitos sofridos pelos giroscópios são minúsculos. Isso implica não só em uma grande conquista no campo da Física, em especial, com profundas implicações na astrofísica, como também em uma motivação para os físicos teóricos adeptos à relatividade geral de Einstein. Contestações futuras da relatividade geral, necessariamente, terão que apresentar resultados superiores aos apresentados pela Sonda Gravidade B.

\section{Referências}

BARKER, B. M.; O'CORNNEL, R. F. Effect of the Earth's Revolution Around the Sun on the Proposed Gyroscope Test of the Lense-Thirring Effect. Physical Review Letters, v. 25, 21, p. 1511-1512, 1970.

CASTINEIRAS, J.; CRISPINO, L. C. B. Relatividade Geral: fundamentos e primeira comprovação experimental. Ciência e Cultura. v. 71. n. 3, p 16 - 22 ,2019

CIUFOLINI, I.; WHEELER, J. A. Gravitation and Inertia. New Jersey: Princeton Series in Physics, 1995.

COHEN, J. M. Note on the Kerr Metric and Rotating Masses. Journal of Mathematical Physics, v. 8, 7, p. 1477-1478, 1967.

EVERITT, C. W. F et al. Gravity Probe B Data Analysis. Space Sci Rev, v. 148, p. 53-69, 2009. 


\section{A Relatividade Geral e a Sonda Gravidade B}

EVERIT'T, C. W. F. et. al. Gravity Probe B: Final Results of a Space Experiment to Test

General Relativity\}. Physical Review Letters, v. 106, 221101, p. 1 - 5, 2011.

FALCIANO, F. T. Geometria, Espaço-tempo e Gravitação: Conexão entre Conceitos da

Relatividade Geral. Revista Brasileira de Ensino de Física, 31, 4, 2009.

HARTLE, J. B. Gravity: An Introduction to Einstein's General Relativity. New York:

Addison Wesley, 2003.

IORIO, L. et. al. Phenomenology of the Lense-Thirring Effect in the Solar System.

Astrophys Space Sci, 331, p. 351 - 394, 2011.

KEPLER, S.O.; SARAIVA, M. F. O. Astronomia e Astrofísica. 2 ed. São Paulo: Livraria da

Física, 2004

KERR, R. P. Gravitational Field of a Spinning Mass as an Example of Algebraically

Special Metrics. Physical Review Letters, v. 11, 5, p. 237-238, 1963.

KRAMER, D. et. al. Exact Solutions of Einstein's Field Equations. New York: Camdridge

University Press, 1980.

LENSE, C. H.; POMPEIA, P. J.; STUDART, N. A deflexão gravitacional da luz: de Newton a

Einstein. Revista Brasileira de Ensino de Física, v. 41. 2019

LETELIER, P. S. Soluções Exatas das Equações de Einstein com Simetria Axial. Revista

Brasileira de Ensino de Física, v. 27, n.1, 2005.

MALEKOLKALAMI, B.; FARHOUDI, M. About Gravitomagnetism. Modern Physics Letters A v. 24 ,p. 601-613, 2009.

MASHHOON, B.; SINGH, D. Dynamics of Extended Spinning Masses in a Gravitational

Field. Physical Review D, v. 74, 124006 p. 1 - 12, 2006.

RUGGiERO, M. L. ; TARTAGLIA, A. Gravitomagnetic Effects. Nuovo Cimento B, v. 117, p. 743-768, 2002.

SHAPIRO, I. et al. Measurement of de Sitter Precession of the Moon: A Relativity Three- 


\section{A Relatividade Geral e a Sonda Gravidade B}

Body Effect. Physical Review Letters, v. 61, p. 2643-2646, 1988.

STELLA, L.; POSSENTI, A. Lense-Thirring Precession in the Astrophysical Context.

Space Science Reviews, v. 148, p. 105-121, 2010.

TARTAGLIA, A.; RUGGIERO, M. L. Gravito-electromagnetism versus Electromagnetism

.Journal of Physics, v. 25, p. 203-210, 2004.

TSOUBELIS, D.; ECONOMOU, A.; STOGHIANIDIS, E. Local and global gravitomagnetic

effects in Kerr spacetime. Physical Review D, v. 36, 4, p. 1045 - 1052, 1987.

VESSOT, R. F. C. et. al. Test of Relativistic Gravitation with a Space-Borne Hydrogen

Maser\}. Physical Review Letters, v. 45, 26, p. 2081-2085, 1980.

WILL, C. M. Finally, Results from Gravity Probe B. Physics, v. 4, 43, 2011.

WILLIAMS, J. G.; NEWHALL, X. X.; DICKEY, J. O. Relativity Parameters Determined from Lunar Laser Ranging. Physical Review D, v. 53, 12, p. 6730-6739, 1996.

Submetido em 23 de novembro de 2020

Aceito em 15 de janeiro de 2021

Publicado em 8 de julho de 2021 